\title{
Ion-Neutral Coupling in Solar Prominences
}

\author{
Holly Gilbert
}

NASA Goddard Space Flight Center, Code 670, Greenbelt, MD 20771

\begin{abstract}
Interactions between ions and neutrals in a partially ionized plasma are important throughout heliophysics, including near the solar surface in prominences. Understanding how ionneutral coupling affects formation, support, structure, and dynamics of prominences will advance our physical understanding of magnetized systems involving a transition from a weakly ionized dense gas to a fully ionized tenuous plasma. We address the fundamental physics of prominence support, which is normally described in terms of a magnetic force on the prominence plasma that balances the solar gravitational force, and the implications for observations. Because the prominence plasma is only partially ionized, it is necessary to consider the support of the both the ionized and neutral components. Support of the neutrals is accomplished through a frictional interaction between the neutral and ionized components of the plasma, and its efficacy depends strongly on the degree of ionization of the plasma. More specifically, the frictional force is proportional to the relative flow of neutral and ion species, and for a sufficiently weakly ionized plasma, this flow must be relatively large to produce a frictional force that balances gravity. A large relative flow, of course, implies significant draining of neutral particles from the prominence. We evaluate the importance of this draining effect for a hydrogen-helium plasma, and consider the observational evidence for cross-field diffusion of neutral prominence material.
\end{abstract}

Keywords: Prominences, filaments, abundances, diffusion

PACS: 52: 52.20Hv, 52.25.Ya, 52.30.Ex

\section{INTRODUCTION}

Coupling between ions and neutrals in magnetized plasmas is fundamentally important to many aspects of heliophysics, including our ionosphere, the solar chromosphere, the solar wind interaction with planetary atmospheres, and the interface between the heliosphere and the interstellar medium. Ion-neutral coupling also plays a major role in the physics of solar prominences, which are referred to as filaments when viewed against the solar disk (we will use the term prominence for both disk and limb phenomena). Prominences are always found in so-called filament channels along polarity inversion lines (PILs), where the magnetic field is highly nonpotential. Filament channels are the origin of all major solar eruptions (coronal mass ejections and eruptive flares), and hence are the subject of intense investigation by the heliophysics community. The temperature of non-erupting filaments is typically on the order of $10^{4} \mathrm{~K}$, and they often appear as a sheet of plasma with a long horizontal dimension (length $\sim 10^{5} \mathrm{~km}$, width $\sim 10^{4} \mathrm{~km}$ ). Although the exact abundance is currently unknown, prominences are composed primarily of helium and hydrogen. Often it is assumed that prominences are composed of $10 \%$ helium and $90 \%$ hydrogen by number since the typical cosmic abundance of helium is $10 \%$. 
Although the basic properties of prominences have been determined and studied for many years, the nature and importance of those basic properties in the context of solar eruptions is poorly understood. Of particular importance are prominence support, structure, and dynamics and the relationship between them. Several different models have been proposed to explain how cool, dense objects like prominences can be supported in and thermally isolated from the surrounding hot, tenuous coronal plasma (e.g., Tandberg-Hanssen 1995), and these models generally fall into one of two main categories: dip models (Kippenhahn \& Schlüter 1957, Antiochos \& Klimchuk 1991), and flux rope models (Hirayama 1985, Leroy 1989, Kuperus \& Raadu 1974, Pneuman 1983, Anzer 1989). The commonality of these two types of solar prominence models lies in the existence of concave-upward directed magnetic fields to support the prominence plasma against the downward gravitational force. Implicit in such support mechanisms is the assumption that the prominence plasma is frozen to the magnetic field lines. The prominence plasma, however, is only partially ionized, which leads to the question of how the neutral component of the plasma is supported and how rapidly neutral material might drain across field lines. The initial steps toward a better understanding of how ion-neutral coupling impacts prominence support, structure and dynamics are presented here: first, an investigation into how quickly neutrals drain across magnetic field lines and out of prominences (§2), and lastly, observations supporting the detection of the large difference in loss timescales between $\mathrm{H}$ and $\mathrm{He}$ (§3).

\section{NEUTRAL ATOM DIFFUSION- CALCULATIONS}

To address the question of how the neutral component of prominence plasma is supported in dip and flux rope models, Gilbert et al. (2002) invoked a simple geometry (simpler than the flux-rope and dip models) in which the magnetic field, $\mathbf{B}$, is in the $\mathrm{x}$ direction and the gravitational acceleration, $\mathbf{g}$, is in the negative $z$-direction, and assumed no flow along the magnetic field lines (Fig. 1).

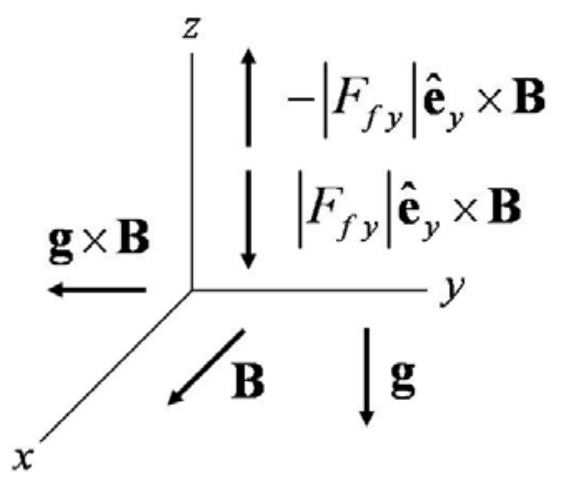

FIGURE 1. Coordinate system showing the simplified prominence magnetic field geometry assumed in the calculations of Gilbert et al. (2002), together with directions of secondary drifts in the y-direction and the tertiary drifts in the positive and negative z-directions. From Gilbert et al. (2002).

In this simple geometry, the ions in the prominence plasma drift in the negative $y$ direction (in the direction of $\mathbf{g} \times \mathbf{B}$ ), while the electrons drift in the opposite direction. 
These drifts produce a current density, $\mathbf{J}$, and a consequent $\mathbf{J} \times \mathbf{B}$ force that just balances the downward gravitational force on the ions and electrons. The neutral atoms in the partially ionized prominence plasma do not sense the magnetic field, and thus fall downward in the direction of the gravitational force. The downward flow of neutral atoms relative to the ionized components of the plasma creates frictional forces between the two components: specifically, the ions and electrons exert upward frictional forces on the neutrals, and the neutrals exert equal and opposite downward frictional forces on the ions and electrons. The downward frictional force on the ions and electrons enhances the drift current produced by the downward gravitational force. Under the condition of equilibrium momentum balance, which is equivalent to force balance, the net drift current is such that the upward $\mathbf{J} \times \mathbf{B}$ force just balances the total downward gravitational force on the entire (i.e., both ionized and neutral components) prominence plasma.

The basic physics underlying support of a partially ionized prominence is relatively simple, as discussed above, but in studying an $\mathrm{H}$-He prominence plasma five particle species have to be considered: e, $\mathrm{H}^{+}, \mathrm{He}^{+}, \mathrm{H}$, and He. Gilbert et al. (2002) calculated the loss time scales for $\mathrm{H}$ and He arising from downward flow of neutral prominence material across the magnetic field lines by considering the equations derived from momentum balance for a multi-constituent plasma. To keep the problem physically simple the density and temperature are taken to be constant throughout the system. Therefore, mass balance or energy balance need not be considered. The equation for momentum balance for the $j^{\text {th }}$ particle species of a multi-component plasma is shown in Fig. 2. Several assumptions are made (discussed in detail in Gilbert et al. 2002) allowing the equation to be greatly simplified.

$$
\begin{aligned}
& \text { General Momentum Balance Equation ( } j^{\text {th }} \text { particle species) } \\
& \frac{\partial}{\partial t}\left(\rho_{j} \mathbf{u}_{j}\right)+\nabla \cdot\left(\rho_{j} \mathbf{u}_{j} \mathbf{u}_{i}\right)=-\nabla \mathscr{R}_{\alpha}-\nabla \tilde{\tau}_{\alpha}+n_{j} e Z_{j}\left(\mathbf{K}+\mathbf{u}_{j} \times \mathbf{B}\right)-\rho_{j} \frac{G M_{\square}}{r^{2}} \hat{\mathbf{e}}_{r} \\
& +\rho_{j} \sum_{k} v_{j k}\left(\mathbf{u}_{k}-\mathbf{u}_{j}\right)+W_{R_{j}}+F_{\% j}+m_{j}\left[\sum_{k} \rho_{j k} \mathbf{u}_{k}-\mathcal{L}_{j} \mathbf{u}_{j}\right] \\
& \text { EXAMPLE: Proton Force Balance } \quad\left(\mathbf{B}=B \hat{\mathbf{e}}_{\mathrm{x}}, \mathbf{g}=-\hat{\mathbf{e}}_{\mathrm{z}} G M / \mathbf{r}^{2}\right) \\
& \text { z-component: } u_{p y}=-g / \Omega_{p}+\Omega_{p}^{-1}\left[v_{p e}\left(u_{e z}-u_{p z}\right)+v_{p H}\left(u_{H z}-u_{p z}\right)\right. \\
& \left.+v_{p H e}\left(u_{H e z}-u_{p z}\right)+v_{p H e^{+}}\left(u_{H e^{+} z}-u_{p z}\right)\right] \\
& \text { y-component: } \quad u_{p z}=\Omega_{p}^{-1}\left[v_{p e}\left(u_{e y}-u_{p y}\right)+v_{p H}\left(u_{H y}-u_{p y}\right)\right. \\
& \left.+v_{p H e}\left(u_{H e y}-u_{p y}\right)+v_{p H e^{+}}\left(u_{H e^{+} y}-u_{p y}\right)\right]
\end{aligned}
$$

FIGURE 2. Equation for momentum balance for the $j^{\text {th }}$ particle species of a multicomponent plasma (simplified by neglecting a number of terms) and an example of the resulting $\mathrm{z}$ and $\mathrm{y}$ components of force balance for protons.

The momentum balance for a five-component plasma gives ten equations in ten unknowns to describe the flow of the components perpendicular to the magnetic field (see Appendix A of Gilbert et al. 2002). Actually, these can be thought of as force 
balance equations, since those terms involving the time dependence of the momentum density and the divergence of the momentum flux density have been neglected. In these equations, the frictional force term serves to couple all five particle species (as illustrated in the bottom part of Fig. 2), while the magnetic force term couples flow in the two directions perpendicular to the magnetic field for ions and electrons.

Figure 3 provides a qualitative pictorial representation of the results of the calculations. The only really large velocities are seen to be associated with the downflow of $\mathrm{H}$ and $\mathrm{He}$. The $\mathrm{y}$-component velocities are what we expect from our drift arguments for the ions and electrons and from the frictional force balance for neutrals. The vertical ion and electron velocities at first seem odd, with the $\mathrm{He}^{+}$and electron velocities downward and the $\mathrm{H}^{+}$velocity upward. But these velocities can be understood as arising from the vertical ion and electron drifts resulting from horizontal frictional forces. The $\mathrm{He}^{+}$ions have the largest velocity in the $-\mathrm{y}$-direction, so the frictional force they experience is in the +y-direction, leading to a downward $\mathbf{F} \times \mathbf{B}$ drift, while the electrons have the largest velocity in the $+y$-direction, so the frictional force they experience is in the -y-direction, leading to a downward $\mathbf{F} \times \mathbf{B}$ drift. Finally, the net frictional force on the protons (for the parameters considered) is in the $-\mathrm{y}$-direction, leading to an upward $\mathbf{F} \times \mathbf{B}$ drift.
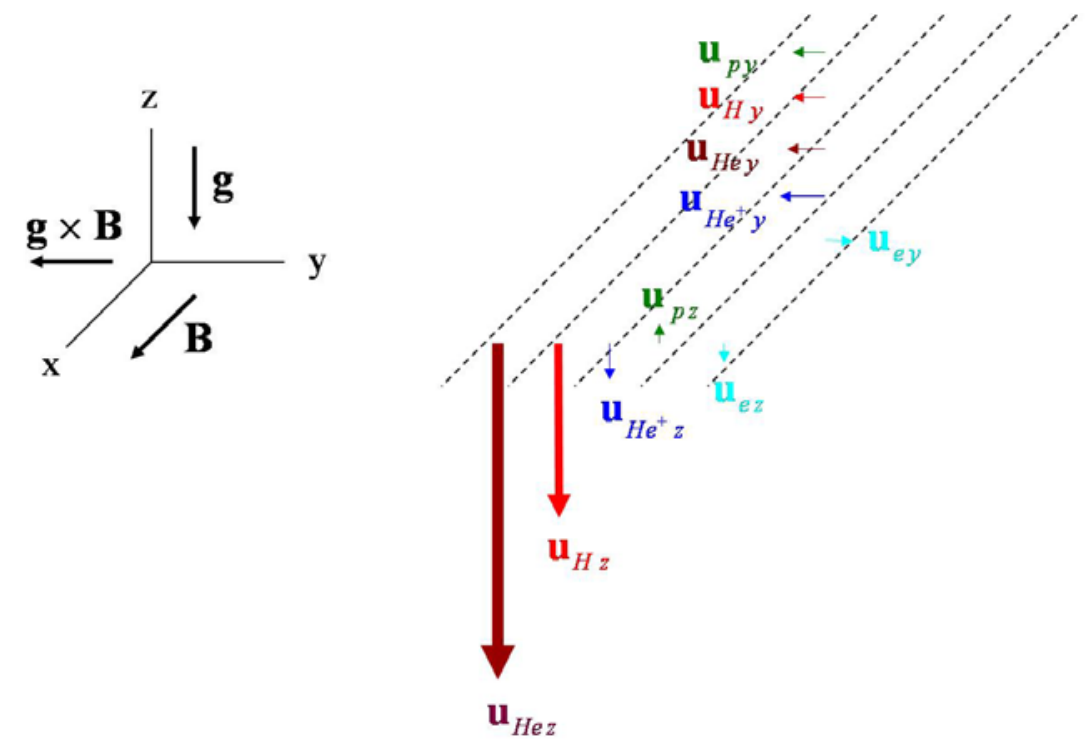

FIGURE 3. Schematic illustration of the directions and relative magnitudes of the vertical and horizontal flow velocities of each of the five particle species. From Gilbert et al. (2002).

For nominal values of prominence height and density, it was found that the crossfield vertical draining times for $\mathrm{H}$ and $\mathrm{He}$ are 520 hours and 24 hours, respectively, and that these times are directly proportional to the vertical column density $\left(n h_{\text {prom }}\right)$ :

$$
\tau_{\mathrm{H}} \approx 520\left[\frac{h_{\text {prom }}\left(\mathrm{R}_{\text {sun }}\right)}{0.01 \mathrm{R}_{\text {sun }}}\right]\left[\frac{n\left(\mathrm{~cm}^{-3}\right)}{10^{10} \mathrm{~cm}^{-3}}\right] \text { hours }
$$




$$
\tau_{\mathrm{He}} \approx 24\left[\frac{h_{\text {prom }}\left(\mathrm{R}_{\text {sun }}\right)}{0.01 \mathrm{R}_{\text {sun }}}\right]\left[\frac{n\left(\mathrm{~cm}^{-3}\right)}{10^{10} \mathrm{~cm}^{-3}}\right] \text { hours }
$$

Here, $h_{\text {prom }}$ is the vertical extent (i.e., height) of the prominence, and $n$ is the number density of the respective species in the prominence. Cross-field draining can play a significant role in modifying the relative absorption ratio, $\frac{\mathrm{He} \mathrm{I}}{\mathrm{H} \mathrm{I}}$, (throughout the rest of the paper, we refer to the ratio, $\frac{\mathrm{He} I}{\mathrm{H} \mathrm{I}}$, simply as the "absorption ratio”, or the $\mathrm{He} / \mathrm{H}$ ratio) if a typical prominence does not undergo significant thermal or dynamical evolution over a period of several hours. In a cursory examination of co-temporal $\mathrm{H} \alpha$ and He I full disk images obtained at HAO’s Mauna Loa Solar Observatory (MLSO), Gilbert et al. (2002) found possible indications of such abundance effects. Motivated by the preliminary indications of varying $\mathrm{He} / \mathrm{H}$ ratio in filament observations, Gilbert et al. (2007) conducted a thorough quantitative study that examines the temporal and spatial variation of the absorption ratio in a sample of quiescent filaments of varying size.

\section{NEUTRAL ATOM DIFFUSION- OBSERVATIONAL SUPPORT}

Investigating the temporal and spatial variation of the relative abundance of He to $\mathrm{H}$ in a sample of solar filaments by comparing co-temporal observations of $\mathrm{H} \alpha$ and $\mathrm{He}$ I (10830 A) provides insight into the importance of cross-field diffusion of neutrals as a potential mass loss mechanism in prominences. The data used in the study conducted by Gilbert et al. (2007) was collected at MLSO, which operates daily (weather permitting) collecting data for about 9 hours a day (typically 17:00-02:00 UT), producing some 180 images from each of its instruments. The two instruments used, both operating with a 3-minute temporal cadence, are the Polarimeter for Inner Coronal Studies (PICS) $\mathrm{H} \alpha$ instrument and the Chromospheric Helium Imaging Photometer (CHIP), which observes in the He I (10830 $\AA$ ) spectral line (see Gilbert et al. 2007 for more details).

The filaments in this study were chosen to encompass a range of sizes and locations, but the main focus is on relatively stable filaments that are visible over a majority of one disk passage. Such filaments allow an examination of the temporal variation of the relative absorption ratio over a relatively long time period. It is worth noting we do not obtain an absolute absorption ratio since the formation processes of the two spectral lines are different. When observed across an entire disk passage, it was found that a majority of stable, quiescent filaments show a relative helium deficit in the upper portions of their structure coupled with a relative helium surplus in the lower regions (see Fig. 4), a consequence of the large loss time scale for neutral hydrogen compared to neutral helium. We refer to this phenomenon as "edge effects", which simply describe the obvious dark bands (representing a relative helium deficit) in the absorption ratio images. 

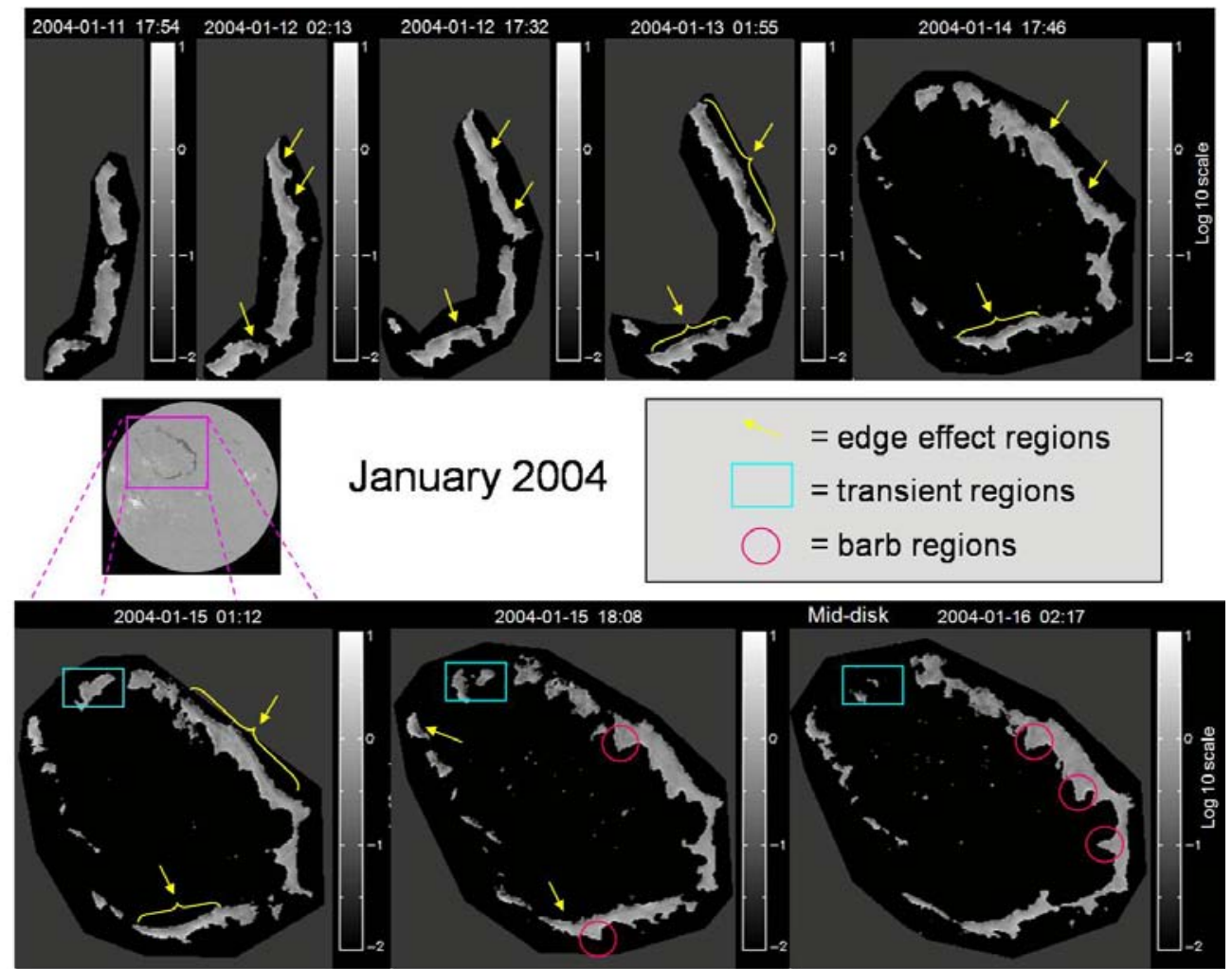

FIGURE 4. Series of images showing absorption ratios, He/H, for a large/stable filament observed in 2004 January. The images are produced such that the color represents the absorption ratio in a logarithmic scale, $\log (\mathrm{He} \mathrm{I} / \mathrm{H} \alpha)$, and a darker pixel correlates to a deficit of absorption in $\mathrm{He} \mathrm{I}$ as compared to $\mathrm{H} \alpha$, while a brighter pixel has a relative surplus of helium. From Gilbert et al. (2007).

Both neutral hydrogen and helium draining time scales are proportional to the vertical total atom column density of the prominence (Gilbert 2002), suggesting that those prominences with larger vertical column densities will drain their neutral material more slowly than those with small column densities and the effects of crossfield diffusion of neutrals should be noticeable when comparing He I (10830 $\AA)$ and $\mathrm{H} \alpha$ data if the time scale for diffusion is much shorter than the dynamical time scale. These results also have interesting observational implications for the distribution of neutral material within filaments. The variation in height due to the filament geometry results in an array of possible signatures with a couple of examples shown in Figs. 5 and 6. Since the loss time scales are proportional to the vertical column density, the $\mathrm{H}$ and He should drain from the sides much more rapidly than from the center for the simple geometry in the Fig. 5: the relative $\mathrm{H}$ to He draining time still remains large. Moreover, the timescales for draining will vary with height in the filament since the highest part of the filament does not have He draining into it from above. The replacement of He from above in the lower portions of the filament leads to an increase in the draining time for that portion. These two effects result in a much shorter time scale for loss of neutral material in the sides of the filament relative to the center in Fig. 5, so we expect to detect a relative He deficit at the sides, or at the edges, in the observations of disk center filaments with simple cylindrical geometries. We make a similar argument for filaments located closer to the limb; when observing a 
filament far from disk center with the same simple geometry, one edge in the observations represents the top of the filament (where we expect a significant He deficit) and the other, the bottom (relative He surplus). A filament located at intermediate longitudes would exhibit a mixed signature, leading to some He deficit at both edges. Consider a more realistic quiescent prominence geometry in which the vertical extent can be much larger than the width, creating a ribbon-like shape (Fig. 6).

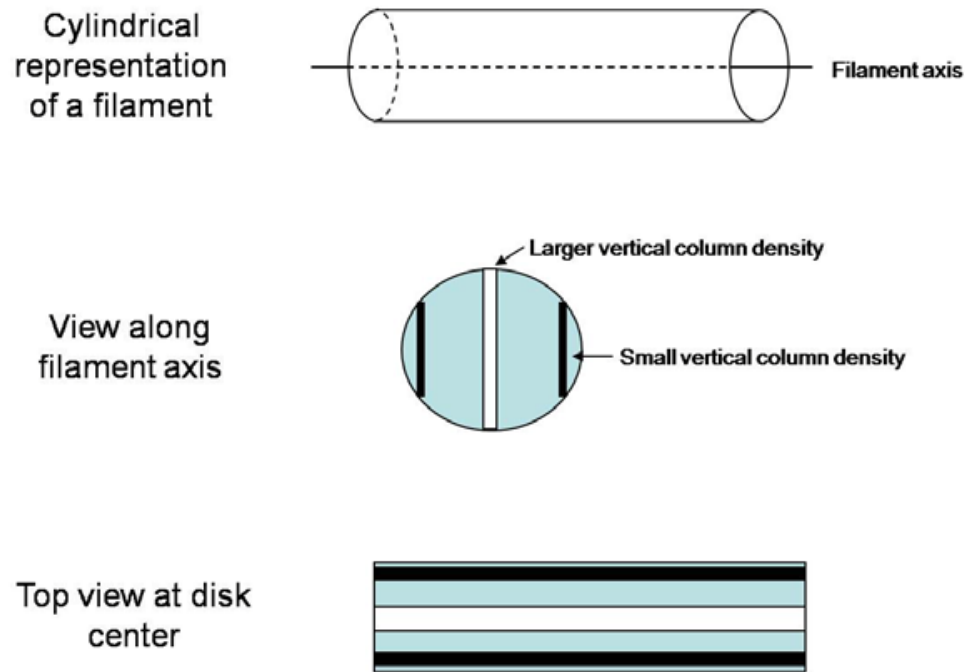

FIGURE 5. Cylindrical filament geometry with a cicular cross section in which the edges represent the sides of the filament, and the height, or equivalently the vertical column density, at the sides is small in comparison with the height and vertical column density at the center. From Gilbert et al. (2007).

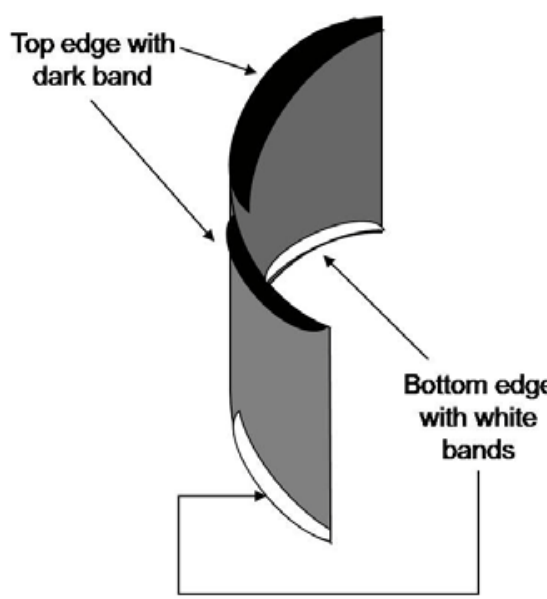

a)

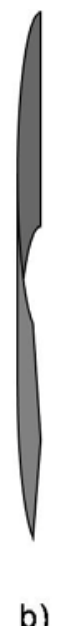

b)

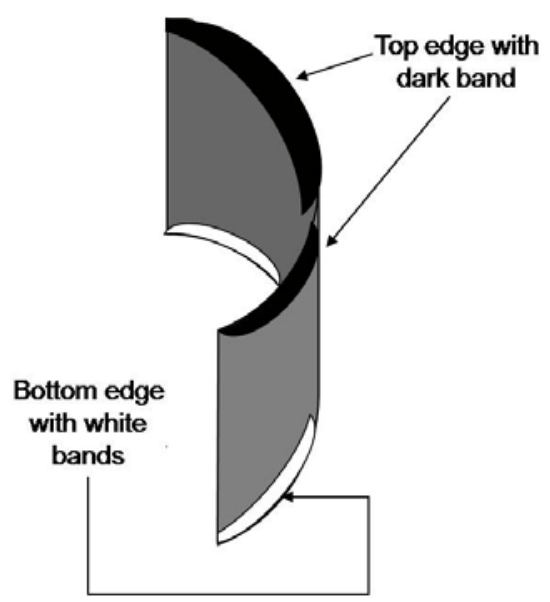

c)

FIGURE 6. Cartoon representing a north-south oriented filament with extreme ribbon-like geometry in which the vertical extent is much larger than the horizontal extent located near (a) the east limb, (b) disk center, and (c) the west limb. From Gilbert et al. (2007).

In this geometry we might expect to see relative helium deficits along the edges in the filaments located away from disk center, since one edge should generally be observed sitting higher in the atmosphere than the other edge (Fig. 6a \& c). At disk center this division might not be apparent since the line-of-sight involves looking 
directly down through the axis of the ribbon. In this orientation, our line of sight is an integration from the very top through the entire depth of the filament, making it impossible to distinguish any region of relative helium deficit from one of relative helium abundance. However, due to the rapid draining of He from the top of a filament relative to its bottom, we expect to see an observational signature of crossfield diffusion at some point during the passage of a filament across the solar disk, regardless of geometry. This picture is consistent with the resulting observations (e.g., Fig. 4). These observations rule out all other causes of $\mathrm{He} / \mathrm{H}$ variations other than draining across field lines and thus give a real observational test of the mechanisms proposed in Gilbert et al. (2002).

\section{DISCUSSION}

A number of different mechanisms have been proposed to drive the mass evolution in prominences, typically resulting in either draining or loading of mass in the prominence magnetic system. Mechanisms responsible for pre-eruption mass loss can involve flow either along field lines, or flow across field lines, driven by MHD instabilities or the cross-field diffusion of neutral material. In this paper we have summarized the evidence that cross-field diffusion of neutrals is a mass loss mechanism in prominences. The next step in understanding how ion-neutral coupling affects prominence formation and evolution is to use a combination of time-dependent multifluid models in 1D, 2.5D, and 3D with high-resolution prominence observations, a project presently underway.

\section{ACKNOWLEDGMENTS}

I wish to thank the co-authors of the studies presented in this work including Thomas Holzer, Viggo Hansteen, Gary Kilper, and David Alexander. I would also like to thank the coronal group at HAO for providing the MLSO data that made this work possible.

\section{REFERENCES}

1. Antiochos, S. K., \& Klimchuk, J. A. 1991, ApJ, 378, 372

2. Anzer, U. 1989, in Dynamics and Structures of Quiescent Solar Prominences, ed. E. R. Priest (Dordrecht: Reidel), 143

3. Elmore, D. F., et al. 1998, Appl. Opt., 37, 4270

4. Gilbert, H. R., Hansteen, V. H., \& Holzer, T. E. 2002, ApJ, 577, 464

5. Gilbert, H. R., G. Kilper, D. Alexander, ApJ, 671, 978, 2007

6. Hirayama, T. 1985, Sol. Phys., 100, 415

7. Kippenhahn, R., \& Schlüter, A. 1957, Z. Astrophys., 43, 36

8. Kuperus, M., \& Raadu, M. A. 1974, A\&A, 31, 189

9. Leroy, J. L. 1989, in Dynamics and Structures of Quiescent Solar Prominences, ed. E. R. Priest (Dordrecht: Reidel), 77

10. Pneuman, G. W. 1983, Sol. Phys., 88219

11. Tandberg-Hanssen, E. 1995, The Nature of Solar Prominences, (Dordrecht: Kluwer)

12. Zirker, J. B, Engvold, O., \& Martin, S. F. 1998, Nature, 396, 440 